\title{
Ruptured Ovarian Pregnancy- A Case Report
}

\author{
Haelom Liegise ${ }^{1}$, Madhu Shweta Sharma ${ }^{2}$, Victor Khuman ${ }^{3}$ \\ ${ }^{1,2}$ Junior resident, \\ ${ }^{3 .}$ Senior resident
}

\begin{abstract}
Ovarian pregnancy accounts for up to 3\% of all ectopic pregnancy but is the most common form of non-tubal ectopic pregnancy. Its diagnosis often is difficult to distinguish as it is frequently confused with a ruptured corpus luteum cyst and clinically with a tubal ectopic. Hereby, presenting a case of primary ovarian pregnancy in a 29-year-old patient, referred from District Hospital Thoubal as query ectopic to Regional Institute of Medical Sciences (RIMS) Imphal. She presented with the complaint of severe lower abdominal pain with history of loss of consciousness, severe pallor and per vaginal findings suggestive of ruptured ectopic.The decision for emergency laparotomy was taken and intraoperatively it was found that patient showing intact tube with ruptured ovarian pregnancy, hence proceeded with right oophorectomy. Aetiological, clinical and therapeutic aspects of this rare extrauterine pregnancy are described. Also, the problems of its differential diagnosis are discussed.
\end{abstract}

Keywords: Rupture, ovarian pregnancy, laparotomy, ultrasonography

\section{Introduction}

Ovarian pregnancy is a rare type of extrauterine pregnancy. Primary ovarian pregnancy is a rare entity, the diagnosis of which continues to challenge the practising clinicians. In contrast to tubal pregnancy, ovarian pregnancy occurs as a single event in otherwise healthy women. There is no specific clinical, laboratory test or ultrasonography signs for differentiating ovarian from tubal pregnancy. Laparoscopy frequently shows haemorrhage from the corpus luteum or a rupture of ovarian cyst. Histology is the only means of establishing the diagnosis. Ovarian pregnancy is often more dangerous than tubal pregnancy, but conservative treatment is often possible.

Here we report a rare case of ruptured ovarian pregnancy whose tissue samples sent to the (pathology) department confirms the presence of products of conception with normal tubal morphology.

\section{Case Report}

29 year old female with G4P2L2A1 was , referred from District Hospital Thoubal to Regional Institute of Medical Sciences(RIMS) Imphal .she had complaints of lower abdominal pain and history of loss of consciousness, she was severely anaemic, acyanotic , afebrile PR 102/ minute ;BP :100/60mmHg;CVS: S1S2 heard ; RS: normal vesicular breath sounds heard ;P/A-distended ,guarding+, tenderness+ ;P/V-uterus -size could not be assessed; right sided fornical fullness positive, left fornix free no cervical motion tenderness. Hb.was $5.6 \mathrm{~g} / \mathrm{dl}$.

She was diagnosed provisionally as ruptured tubal pregnancy and proceeded to emergency laparotomy. Intraoperatively there was hemoperitoneum around $1000 \mathrm{ml}$ with $800 \mathrm{ml}$ clots .Both the tubes found to be normal. Left ovary was normal and right ovary found ruptured possessing products of conception and hence diagnosed as ruptured ectopic ovarian pregnancy.The decision for right oophorectomy was taken and excecuted.Two unit of PRBC was transfused intra operatively.

The tissue sent to pathology department showed products of conception and normal tubal morphology.

Thus, the intra-operative findings and the histopathology examination satisfied the criteria for ovarian pregnancy as described by Spiegelberg $1878,[4]$ which is as follows:

a) intact fallopian tube on the affected side,

b) fetal sac must occupy the position of the ovary on the affected side,

c) ovary connected to the uterus by ovarian ligament,

d) ovarian tissue must be located in the sac wall, which was confirmed by histopathology. 


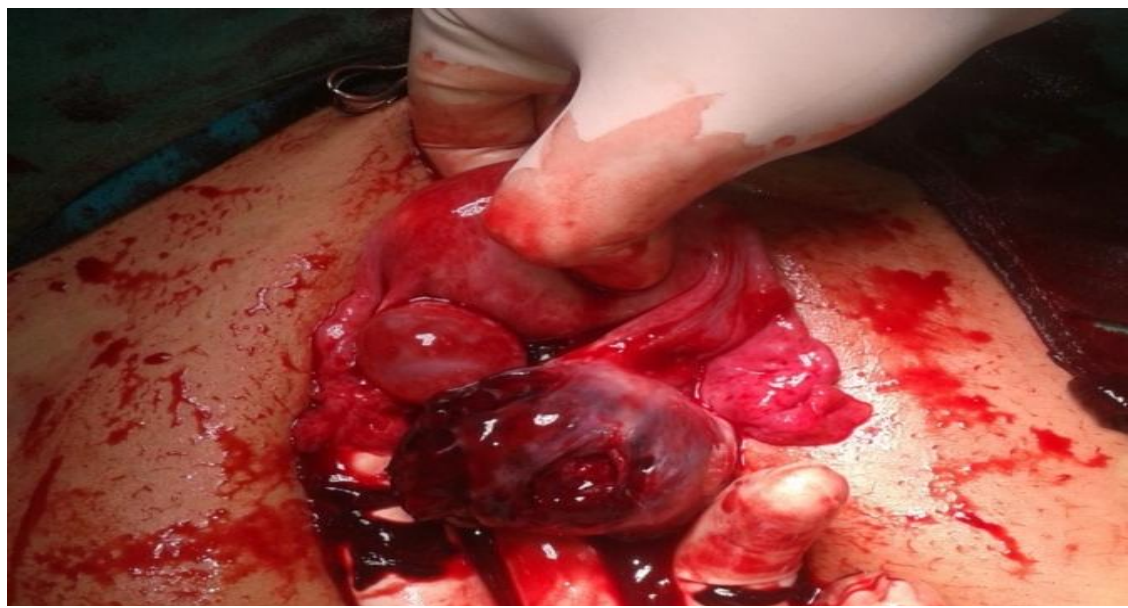

Intraoperatively: Right Ovarian Ectopic
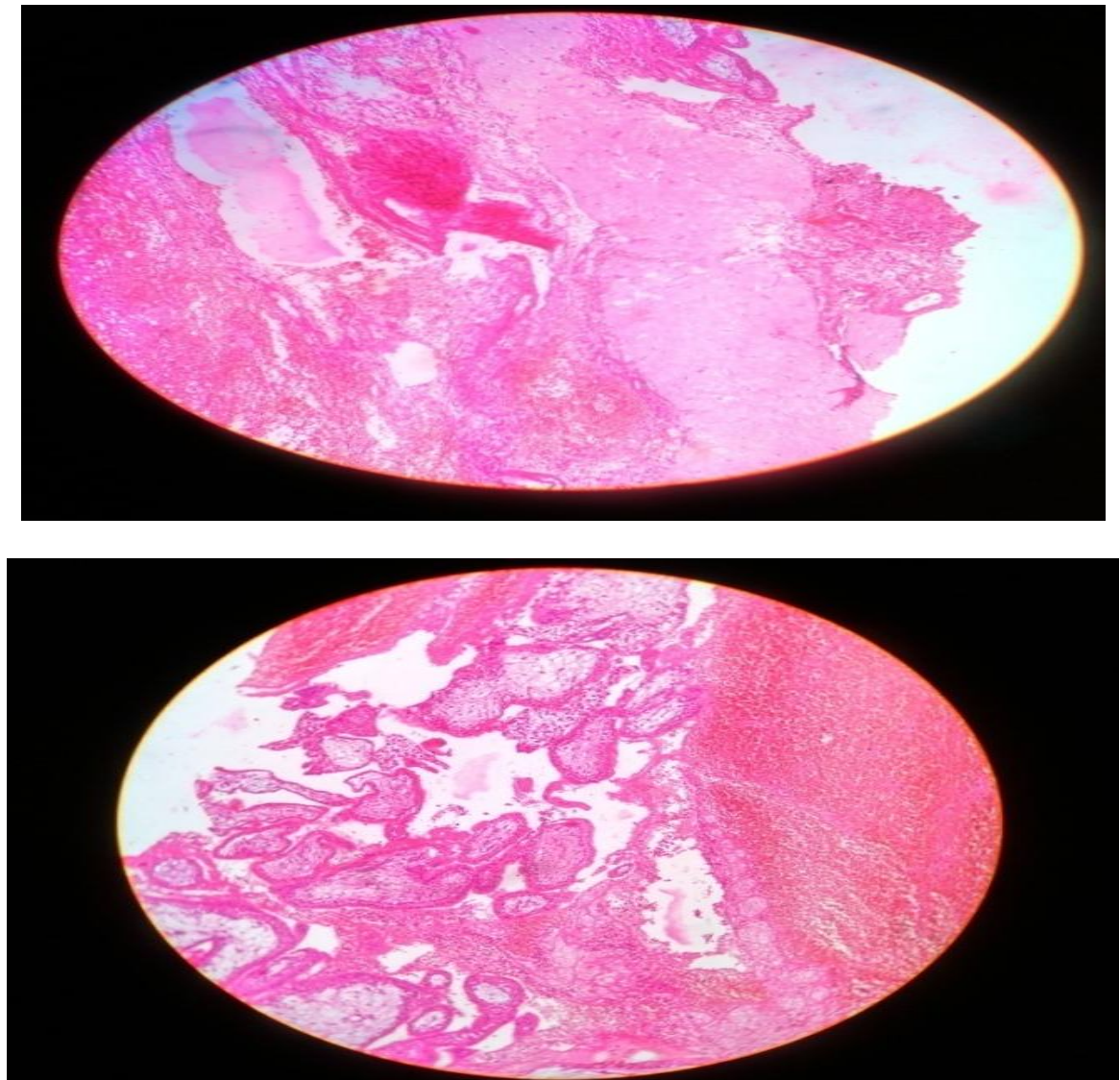

HPE of Right Ovarian Ectopic

\section{Discussion}

Ovarian pregnancies are rare. The vast majority of ectopic pregnancies occur in the fallopian tube; only about $0.15-3 \%$ of ectopics occur in the ovary (1).The incidence has been reported to be about 1:3,000 to 1:7,000 deliveries.(2)

Ovarian pregnancy is a rare variant of ectopic pregnancy. The etiology of ovarian pregnancy is unknown as the usual causative factors - PID, infertility and pelvic surgery - implicated in tubal ectopic pregnancy have no association with ovarian ectopic. Other hypotheses have suggested interference in the release of the ovum from the ruptured follicle, malfunction of the tubes and inflammatory thickening of the tunica albugenia. It cannot be concluded that intrauterine device increases the risk for ectopic ovarian pregnancy. The entity, empty follicle syndrome, where no oocytes are retrieved from the mature ovarian follicles with apparently normal follicular development and estradiol levels, after controlled ovarian hyperstimulation for an assisted reproductive technology cycle, despite repeated aspiration and flushing, can also be a cause for primary ovarian 
pregnancy(3).Early diagnosis of ovarian pregnancy is necessary in order to avoid more serious complications and emergency invasive procedures .However, preoperative diagnosis remains challenging, and it is diagnosed generally during surgery(4). Correct diagnosis of ectopic pregnancy can often be made on the basis of patient history, signs and symptoms, serum beta-hCG levels, and pelvic USG examination.

Oophorectomy was advocated in the past now ovarian cystectomy and/or wedge resection is advised. Successful medical therapy with methotrexate ia also reported. In our case due to haemodynamic instability of the patient decision for oophorectomy was taken. Few cases of laparoscopic treatment in women with hemoperitoneum have been reported.(5)

\section{Conclusion}

Diagnosing an ovarian pregnancy is difficult .Primary ovarian pregnancy may occur without the presence of any classical risk factors for ectopic pregnancy. So with features suggestive of tubal ectopic pregnancy and intra operative finding of normal tubes should alert the obstetrician to suspect ovarian pregnancy.(5) Early diagnosis and prompt treatment can allow for conservative surgery and future fertility of the patient.

\section{References}

[1]. Nwanodi O,Khulpatea N (2006)."The preoperative diagnosis of primary ovarian pregnancy"Natl Med Assoc.98(5):7968,PMC2569290.PMID 16749658.

[2]. Jump upto:Raziel A,schachter M Mordechal E ,Friedler S,Paski M,Ron-EIR.(2004).”Ovarian pregnancy -a -12- year experience of 19 cases in one mutation “.Eur J Obstet Gynecol Reprod Biol (1)114:92-6,doi:10.1016/j. ejogrb.2003.09.038.PMID 15099878

[3]. Qublan H, Tahat Y, Al-Masri A. Primary ovarian pregnancy after the empty follicle syndrome: A case report. J Obstet Gynaecol Res. 2008;34:422-4. [PubMed]

[4]. F.Plotti,A. Di Giovanni,C.Oliva, F.Battaglia and G.plotti”Bilateral ovarian pregnancy after intrauterine insemination and controlled ovarian stimulation,"Fertility and Sterility,vol.90,no.5,pp.2015.e3-2015e5,2008.

[5]. S.Panda,L.M.Darlong,S.Singh, and T.Borah,"Case report of a primary ovarian pregnancyin a primigravida"'.Journal of Huan reproductive sciences,vol 2.NO.2,PP.90-92,2009. 